\title{
Rapid Synthesis of Nanoporous Conformal Coatings via Plasma- Enhanced Sequential Infiltration of a Polymer Template
}

\author{
Yunlong She ${ }^{\dagger}$ Jihyung Lee, ${ }^{\dagger}$ Benjamin T. Diroll, ${ }^{\ddagger}$ B⿺ Byeongdu Lee, ${ }^{\S}$ Samir Aouadi, ${ }^{\dagger}$
} Elena V. Shevchenko, $*,+\infty$ and Diana Berman*, ${ }^{\dagger}$

${ }^{\dagger}$ Materials Science and Engineering Department, University of North Texas, 1155 Union Circle, Denton, Texas 76203, United States

${ }^{\ddagger}$ Center for Nanoscale Materials and ${ }^{\S}$ Advanced Photon Source, Argonne National Laboratory, 9700 S. Cass Avenue, Argonne, Illinois 60439, United States

\section{Supporting Information}

ABSTRACT: Nanoporous conformal coating is an important class of materials for electrocatalysis, water purification, antireflective coatings, etc. Common synthesis methods of porous films often require harsh conditions (high temperature and high plasma power) or specific substrate materials. Here, we report a plasma-enhanced sequential infiltration synthesis (PE SIS) as a new platform toward deposition of nanoporous inorganic films. PE SIS is based on oxygen-plasma-induced rapid conversion of metal precursors selectively adsorbed in a blockcopolymer template. Porosity and thickness of resulting materials can be easily controlled by characteristics of the template. PE SIS is conducted under gentle conditions, and can be applied to a broad range of substrates, including water-sensitive surfaces. PE SIS offers adventurous rapid infiltration with improved ability to obtain highly interconnected porous alumina films with thicknesses up to $5 \mu \mathrm{m}$. We show that full infiltration of the polar domain of the polymer template can be achieved upon initial exposure to TMA, followed by its oxygen-plasma-induced conversion into a functional material. Since different types of plasma (such as oxygen, nitrogen, hydrogen, etc.) induce conversion of a broad range of metal precursors, PE SIS opens a new approach for synthesis of highly porous materials with various elemental compositions and stoichiometries.

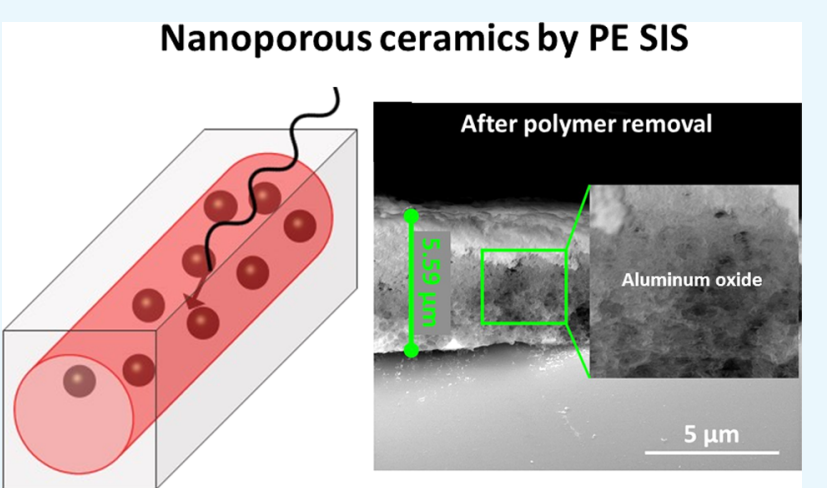

\section{INTRODUCTION}

Water-based thermal atomic layer deposition (ALD) on blockcopolymer $(\mathrm{BCP})$ templates resulted in the discovery of vaporphase self-limiting sequential infiltration synthesis (SIS). ${ }^{1-3}$ SIS based on infiltration of polymer templates with inorganic materials enabled fast growth of nanoporous inorganic structures with finely tuned pitch, thickness, porosity, and composition. The key process of state-of-the art classical thermal ALD and SIS is a reaction of metal precursor chemisorbed inside a polymer template with water vapors. Nanoscale patterning of inorganic materials with tunable characteristic feature size has many potential applications in electronics, ${ }^{4}$ biochemistry, ${ }^{5,6}$ optics, ${ }^{7-9}$ and photonics. ${ }^{10}$ Nanostructured materials with well-controlled morphology over macroscopic length scales have recently enabled the development of high surface area sensors for biomedical applications, ${ }^{11}$ hydrogen storage, ${ }^{12}$ and antireflection coatings for optical applications, ${ }^{13-15}$ as well as friction- and wear-reducing porous surfaces for orthopedic implants ${ }^{16,17}$ and reservoirs for solid lubricants $^{18-20}$ or water filtration. ${ }^{21}$ Porous materials are an important class of nanostructured systems for electrochemical energy storage applications. ${ }^{22}$
Coatings with nanosized features or pores can be fabricated by traditional lithographic approaches. However, the traditional approaches are typically slow and expensive and patterning of features smaller than $50 \mathrm{~nm}$ is rather challenging. ${ }^{23}$ The recently developed SIS is a promising alternative approach for depositing conformal nanostructured inorganic films. This method integrates the ALD process that results in selective infiltration of inorganic precursor in polar domains of the selfassembled block-copolymer templates that reacts with water in the subsequent cycle in a self-limiting regime and leads to the formation of an inorganic structure. ${ }^{24,25}$ In contrast to dense ALD coatings, SIS coatings are nanostructured patterns replicated from the self-assembled block-copolymer templates. The advantage of SIS over ALD is its ability to provide thicker coatings within a few reaction steps. ${ }^{1,26}$ Thermal SIS has been successfully used to improve the lithographic patterning, ${ }^{27}$ design of oil-adsorbing sponges, ${ }^{28}$ and single and multilayer antireflective coatings. ${ }^{29}$ Traditional SIS is based on the reaction between a precursor chemisorbed inside a polymer

Received: September 5, 2017

Accepted: November 1, 2017

Published: November 10, 2017 

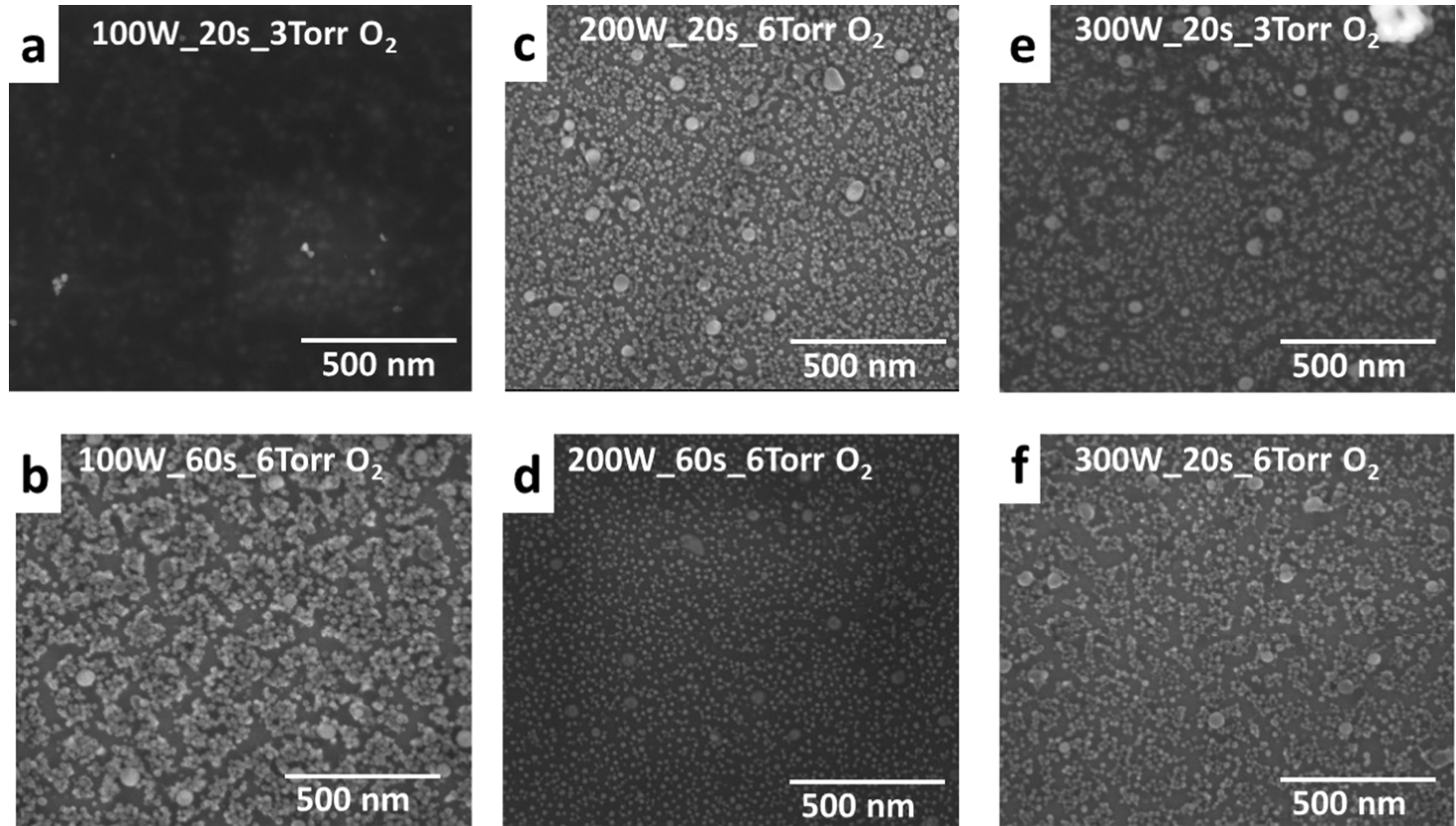

Figure 1. Scanning electron microscopy (SEM) images of porous structures ( $\sim 50 \mathrm{~nm}$ thickness $)$ obtained at different oxygen plasma power exposure times of the nonswelled PS-b-P4VP $(75 \mathrm{k}-b-25 \mathrm{k})$ template infiltrated with TMA to plasma and oxygen pressures. The structures were obtained by five SIS cycles processed as follows: (a) at 3 Torr oxygen, $100 \mathrm{~W}$ plasma for $20 \mathrm{~s}$ of exposure; (b) at 6 Torr oxygen, $100 \mathrm{~W}$ plasma for 60 $\mathrm{s}$ of exposure; (c) at 6 Torr oxygen, $200 \mathrm{~W}$ plasma for $20 \mathrm{~s}$ of exposure; (d) at 6 Torr oxygen, $200 \mathrm{~W}$ plasma for $60 \mathrm{~s}$ of exposure; (e) at 3 Torr oxygen, $300 \mathrm{~W}$ plasma for $20 \mathrm{~s}$ of exposure; (f) at 6 Torr oxygen, $300 \mathrm{~W}$ plasma for $20 \mathrm{~s}$ of exposure.

template and water vapors. However, elevated temperatures required for this reaction in the thermal SIS are incompatible with moisture-sensitive and thermally unstable surfaces or interfaces. Previously, plasma ALD demonstrated advantages over thermal ALD, such as higher quality of the deposited materials, lower impurity content, and better electronic properties; lower deposition temperature; increased choice of precursors and materials; tuned stoichiometry of the compositions as a result of deposition under nonequilibrium conditions; and increased growth rate. ${ }^{30}$ Thus, plasmaenhanced sequential infiltration synthesis (PE SIS) potentially allows the synthesis of a broader range of porous nanostructured materials as compared to thermal SIS and is essential for our ability to design functional materials with advanced properties.

Here, we demonstrate that SIS can be realized via PE conversion of a metal precursor. We show that the exposure of trimethylaluminum (TMA) molecules to oxygen plasma leads to the synthesis of a conformal alumina coating with controlled thickness and porosity. Moreover, we show that the oxygen plasma step of the process facilitates production of fully infiltrated nanoporous structures within one cycle, as compared to a minimum of eight cycles required in water-based thermal $\mathrm{SIS}^{29}$ hence minimizing the time of the synthesis of highly porous structures. We demonstrate that up to $5 \mu \mathrm{m}$ alumina films can be achieved in one step. The alumina films obtained by PE SIS are highly porous, as evidenced by the quenching of photoluminescence (PL) from $\mathrm{CsPbB}_{3}$, upon the exposure of such structures to highly humid environment. In turn, the control sample with $\mathrm{CsPbBr}_{3} \mathrm{NPs}$ protected by solid $10 \mathrm{~nm}$ alumina deposited by PE ALD exhibited photoluminescence 2 orders of magnitude higher after a 5 days of storage in humid environment ( $40 \%$ relative humidity).

\section{RESULTS AND DISCUSSION}

Previous studies demonstrated that alumina can be deposited by thermal ${ }^{31}$ and PE ALD. ${ }^{32,33}$ Thus, we focused our study on synthesis of conformal alumina coatings because it is the most understood system obtained by SIS. Our intent was to explore PE ALD for SIS as an alternative to the thermal ALD approach to produce nanostructured surfaces and coatings with controllable thickness and porosity. PE ALD does not require elevated temperatures, and because the conversion of chemisorbed precursors is achieved by plasma, it suggests potential application to moisture-sensitive and thermally unstable surfaces and interfaces.

First, we concentrated on infiltration of the poly(styreneblock-4-vinylpyridine) (PS-b-P4VP) (75k-b-25k) template with TMA, followed by exposure to oxygen plasma of different powers and duration. The BCP films of $50 \mathrm{~nm}$ were spin-casted on the silicon substrate. After infiltration steps, the residual polymer films were removed using high-temperature annealing $^{29}$ resulted in the formation of porous alumina coatings (Figure 1).

In the case of low power and short exposure of the BCP template to plasma, coatings with low fraction of alumina were obtained (Figure 1a). However, increases of plasma power, exposure time, and pressure facilitated the synthesis of conformal coatings with higher alumina content.

Worth mentioning is that when selecting PE SIS conditions, plasma energy should be high enough to produce the reaction of transformation of all TMA molecules within the polymer template into aluminum oxide species but low enough to avoid damage of the polymer template. The ideal situation for SIS would be if plasma power allows for the growth of the films with subsequent burning of the polymers after complete transformation of the chemisorbed precursors. However, that can be a challenge for BCP. Previous studies on ion etching of 
polymers revealed that the polar part of the polymer molecule is more sensitive to plasma exposure. ${ }^{34,35}$ Thus, a much higher removal rate was reported for polar polymer (e.g., poly(methyl methacrylate) (PMMA)), as compared to almost negligible removal for nonpolar polymer (100\% polystyrene (PS)). ${ }^{34}$ The selective reactivity of TMA precursor with $\mathrm{C}=\mathrm{O}$ and $\mathrm{C}-\mathrm{O}-\mathrm{R}$ functional polar groups of the polymer macromolecule requires minimizing/prevention of polymer damage during plasma exposure while keeping the reactivity of the plasma species high. Therefore, the plasma exposure time during the SIS process is kept short to eliminate/minimize the damage of the polar domains used for templated deposition of inorganic materials in SIS and to prevent generation of stresses in the resulting films, expected to lead to material cracking and detachment.

To understand the effect of oxygen plasma on polar domains in BCP, a Fourier transform infrared (FTIR) study was performed on BCP PS- $b$-P4VP (75k- $b$-25k) (Figure 2) before
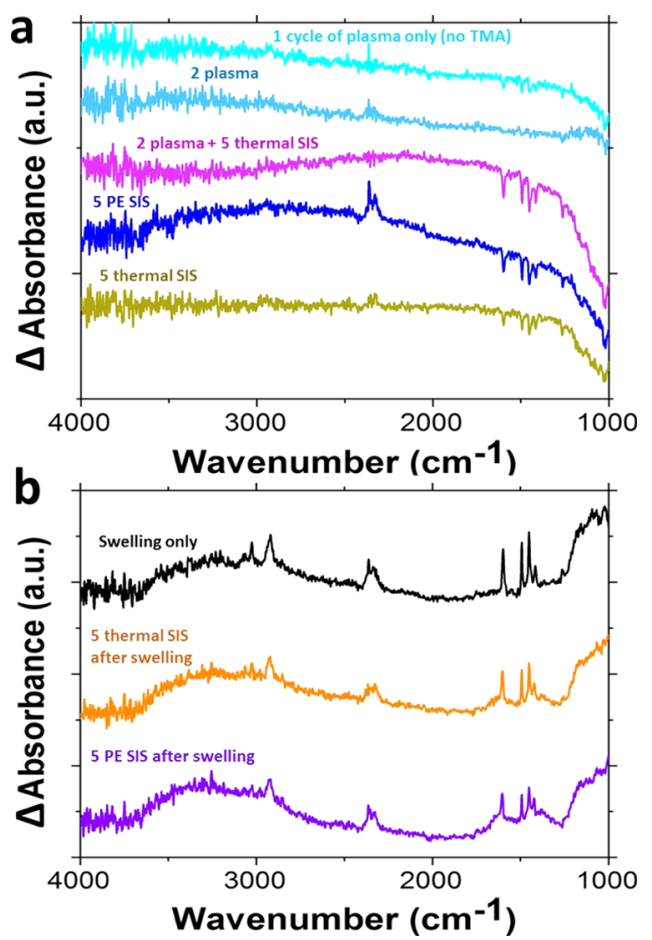

Figure 2. (a) No swelling FTIR delta absorbance measurements for comparison of the effect of thermal SIS and PE SIS when performed on $50 \mathrm{~nm}$ thick PS- $b$-P4VP $(75 \mathrm{k}-b-25 \mathrm{k})$. All of the spectra are referenced to initial as-spin-coated PS- $b$-P4VP. (b) Swelling FTIR absorbance measurements for swelling induced modifications in the polymer during thermal SIS and PE SIS. Swelling was performed in ethanol at $70{ }^{\circ} \mathrm{C}$ for $1 \mathrm{~h}$.

and after PE SIS. Specifically, polymer samples subjected to plasma exposure, swelling, PE SIS, and thermal SIS of various durations were analyzed with FTIR by monitoring the changes in bond configuration of the treated polymer templates as compared to that of the as-spin-coated film.

First, plasma-induced modifications in the polymer films were analyzed. Figure 2a demonstrates no significant changes in the absorbance FTIR spectrum, and only a slight decrease in $\mathrm{C}=\mathrm{O}$ absorbance $\left(\right.$ at $\sim 1729 \mathrm{~cm}^{-1}$ ) and increase in $\mathrm{O}=\mathrm{C}=\mathrm{O}$ absorbance (at $\sim 2350 \mathrm{~cm}^{-1}$ ), when PS- $b$-P4VP is exposed to one or two cycles of plasma. Thus, we can conclude that negligible damage is produced by the plasma in the P4VP domains and that the deposited structure under given plasma conditions can be determined only by the characteristics of the PS- $b$-P4VP template. Second, the alumina infiltration process resulted in a decrease in the absorption for $\mathrm{CH}$ bending of $\mathrm{CH}_{3}$ and $\mathrm{CH}_{2}$ compounds at $1580,1500,1450$, and $1410 \mathrm{~cm}^{-1}$. ${ }^{36}$ This suggests that in the case of $\mathrm{P} 4 \mathrm{VP}$ polymer, $\mathrm{CH}_{x}$ are the major sites for alumina growth.

The same plasma conditions can be applied to other types of $\mathrm{BCP}$, in particular to PS- $b$-PMMA, the most common BCP in SIS, because only minor polymer modifications, such as a slight decrease in $\mathrm{C}=\mathrm{O}$ absorbance of PMMA by plasma, were detected by FTIR. Similar to thermal SIS, ${ }^{24}$ PE SIS on PMMA was accompanied by certain modifications in polymer structures. More specifically, stretching in carbonyl $\mathrm{C}=\mathrm{O}$ (at $\sim 1729 \mathrm{~cm}^{-1}$ ), ester $\mathrm{C}-\mathrm{O}-\mathrm{R}$ ( at $\sim 1145$ and $\sim 1260 \mathrm{~cm}^{-1}$ ), and $\mathrm{C}-\mathrm{H}\left(\right.$ at $\sim 2950 \mathrm{~cm}^{-1}$ ), as well as bending of $\mathrm{C}-\mathrm{H}$ (at $\sim 1450$ $\left.\mathrm{cm}^{-1}\right)^{37,38}$ are observed. A more pronounced decrease in absorbance peaks at 1729,1145 , and $1260 \mathrm{~cm}^{-1}$ in FTIR spectra acquired for five cycles of PE SIS as compared to that in five cycles of thermal SIS is indicative of more rapid consumption of carbonyl and ester groups than that for thermal SIS and therefore more rapid infiltration of PMMA (Figure 3). This observation is in good agreement with the previously observed faster kinetics in PE ALD synthesis of solid conformal coatings of a broad range of materials. ${ }^{32}$

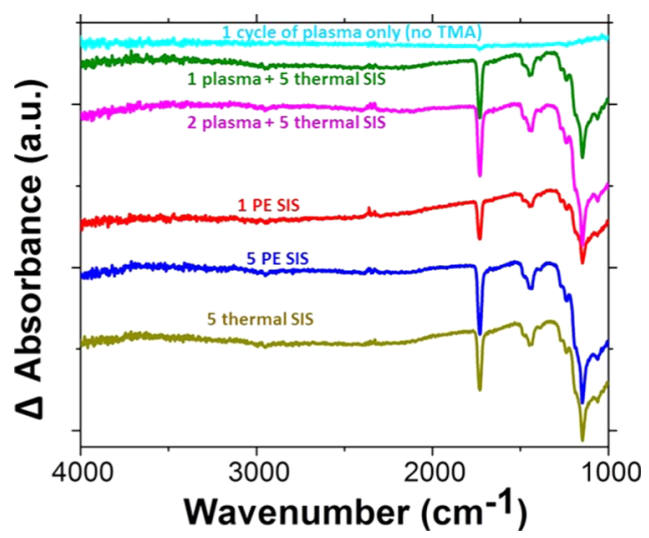

Figure 3. FTIR delta absorbance measurements for comparison of the effect of water-based thermal SIS and PE SIS when infiltrating $50 \mathrm{~nm}$ thick PMMA polymer. All of the spectra are referenced to initial asspin-coated PMMA.

The kinetics of infiltration of BCP templates was studied using grazing incidence small angle X-ray scattering (GISAXS) analysis on samples prepared with one, two, three, and five PE SIS cycles. The two-dimensional GISAXS images for all of the samples are almost identical, one of which is shown in Figure 4a. One-dimensional linecut profiles from the images (Figure $4 \mathrm{~b})$ also revealed that the structures of all four samples show the same form factor oscillation, meaning that the diameters of pores or posts are identical. A minor difference in GISAXS patterns revealed a slightly better resolved structure factor peak at around $q_{\mathrm{y}} \sim 0.02 \AA^{-1}$, as the number of SIS increases (Supporting Information). This suggests more extended inorganic features in the samples with more cycles and can be interpreted by the fact that the infiltration process is fast and likely performed stochastically. One cycle leads to full infiltration of the polar polymer molecule part, with additional 

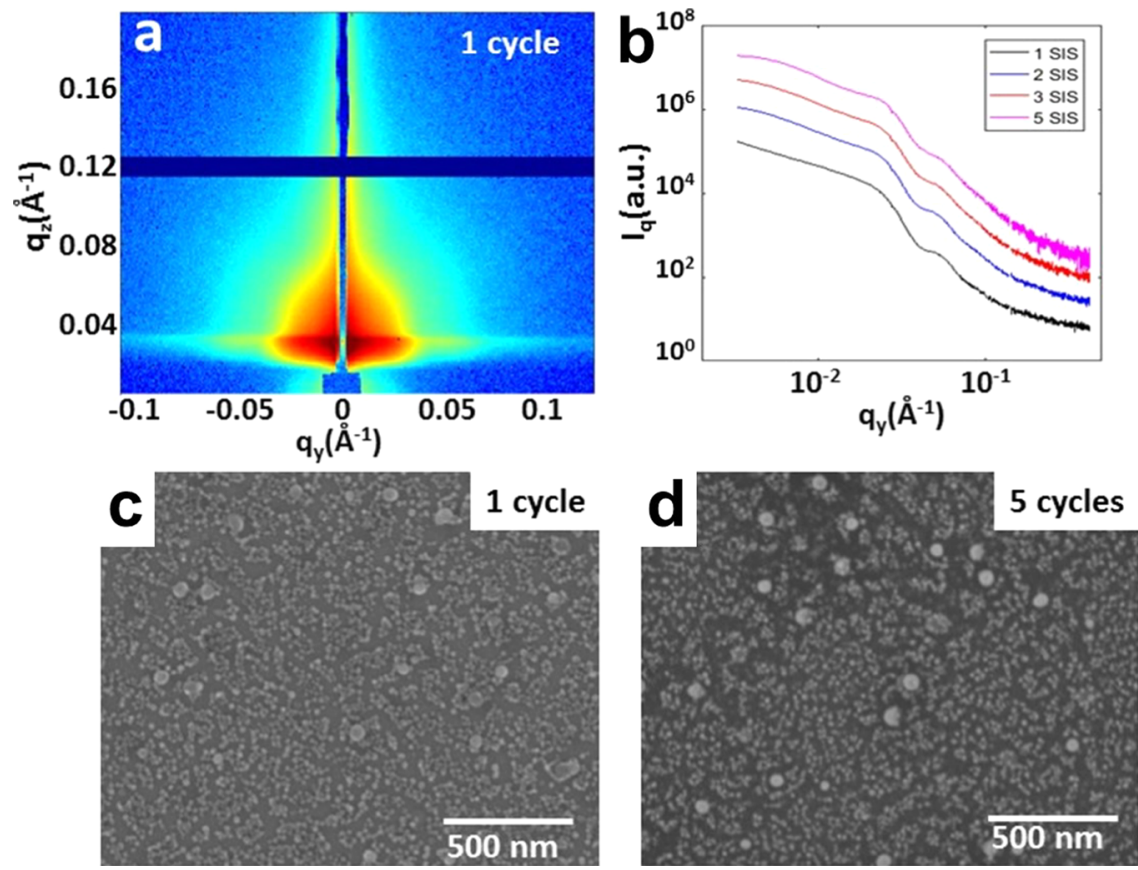

Figure 4. GISAXS analysis of the nonswelled PS-b-P4VP (75k-b-25k) polymer infiltrated with different numbers of PE SIS cycles (300 W, $20 \mathrm{~s}$ of oxygen plasma). GISAXS image of one PE SIS sample (a) and horizontal linecuts (b) of one through five SIS samples are presented. Linecuts are made at $q_{\mathrm{z}} \sim 0.035 \AA^{-1}$ in (a) and scaled arbitrarily for clarity. No noticeable difference in the resulting films deposited with one, two, three, and five PE SIS cycles is observed. SEM images of the films grown with (c) one cycle and with (d) five cycles are included as well. Porosity of the samples is in the $70-80 \%$ range.
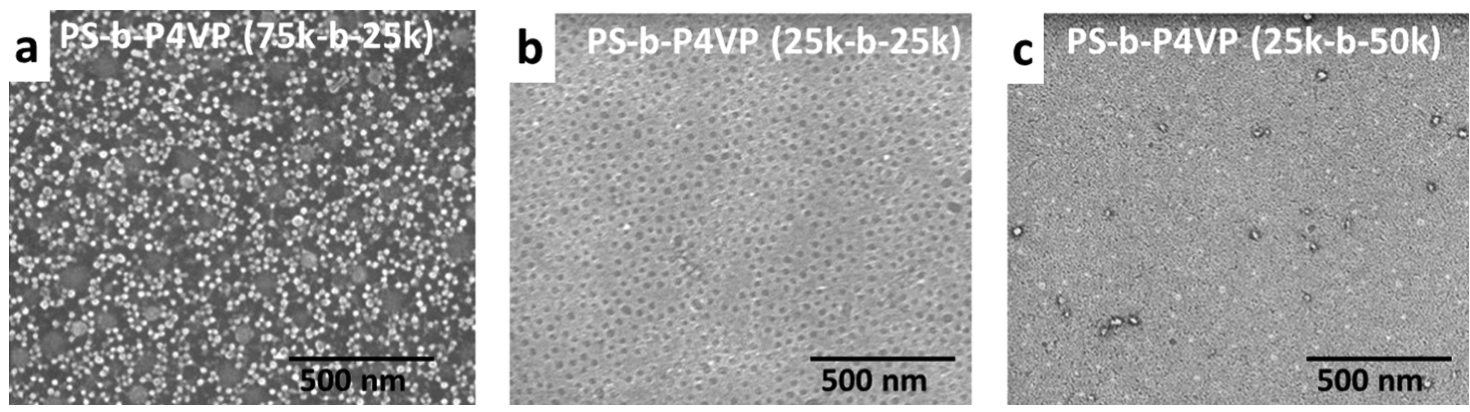

Figure 5. SEM images of porous alumina films grown by the PE SIS infiltration (five SIS cycles) of PS- $b$-P4VP polymers ( $50 \mathrm{~nm}$ thick polymers swelled in ethanol at $70^{\circ} \mathrm{C}$ for $1 \mathrm{~h}$ ) with different volume fraction of polystyrene: (a) $75 \mathrm{k}-b-25 \mathrm{k}$, (b) $25 \mathrm{k}-b-25.5 \mathrm{k}$, and (c) $24 \mathrm{k}-b$-51k. The thickness of the resulting alumina films is in the range of $80-100 \mathrm{~nm}$ with porosity varying from $30 \%$ for $24 \mathrm{k}-b-51 \mathrm{k}$ PS- $b$-P4VP ( $\sim 68: 32$ concentration of polar/nonpolar domains) polymer to 50 and $70 \%$ for $25 \mathrm{k}-b-25.5 \mathrm{k}$ ( $\sim 50: 50$ concentration of polar/nonpolar domains) and $75 \mathrm{k}-b-25 \mathrm{k}(25: 75$ concentration of polar/nonpolar domains) polymers correspondingly.

cycles resulting in growth of materials on top with regular $\sim 1 \AA$ thickness/cycle rate. These results are consistent with reported earlier higher rates of the material's growth in the case of the PE ALD in comparison with the thermal ALD. ${ }^{39}$ Our SAXS results suggest a similar trend for PE SIS as well. Thus, the advantage of PE SIS in terms of eliminating multiple steps of the deposition procedure is demonstrated.

In the case of water-based thermal SIS, we already demonstrated that porosity of the resulting alumina films is highly dependent on the characteristics of the polymer template. $^{29}$ Specifically, the concentration of polar/nonpolar domains in the polymer is one of the major factors determining the resulting porosity of the synthesized aluminum oxide films. The same trend in the effect of polymer composition on the porosity is observed for the PE SIS process (Figure 5). The higher length of the polar part results in a less porous material (Figure 5c). However, as in the case of thermal SIS, control over film thickness was limited by the penetration depth of the TMA. The thickness of the alumina films was in the range of 50-60 nm and did not depend on the thickness of the BCP template. In our previous study, ${ }^{29}$ we demonstrated that the swelling of BCP could be used efficiently to control the thickness and porosity of the deposited films. This was achieved by immersing the PS- $b$-P4VP template into ethanol, ${ }^{40}$ which is selective to the P4VP block, and drying afterwards. The generated additional porosity throughout the film in the positions where the P4VP block was swelled and subsequently collapsed allowed more efficient infiltration of a thick film.

Here, we also implemented the swelling procedure to initiate additional porous channels for TMA infiltration. For this purpose, we spin-coated a thick PS- $b$-P4VP film $(\sim 5 \mu \mathrm{m}$ thickness) on the silicon substrate. The major limiting factors for developing such thick films are uniformity of the spincoated polymer and adhesion to the substrate when performing 

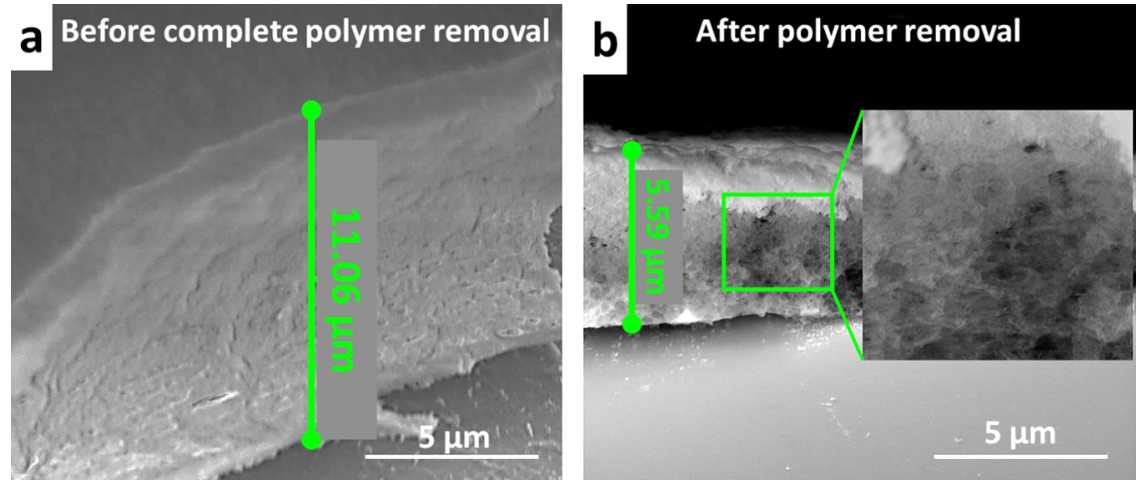

Figure 6. Thick nanoporous films from PS- $b$-P4VP (75k-b-25k) polymer. (a) Before complete polymer removal, the film demonstrates a charging effect and thickness of $\sim 11.1 \pm 1.8 \mu \mathrm{m}$. (b) After complete removal of the polymer by burning, the porous structure is observed and the resulting thickness of the film is $\sim 5.6 \pm 0.4 \mu \mathrm{m}$.
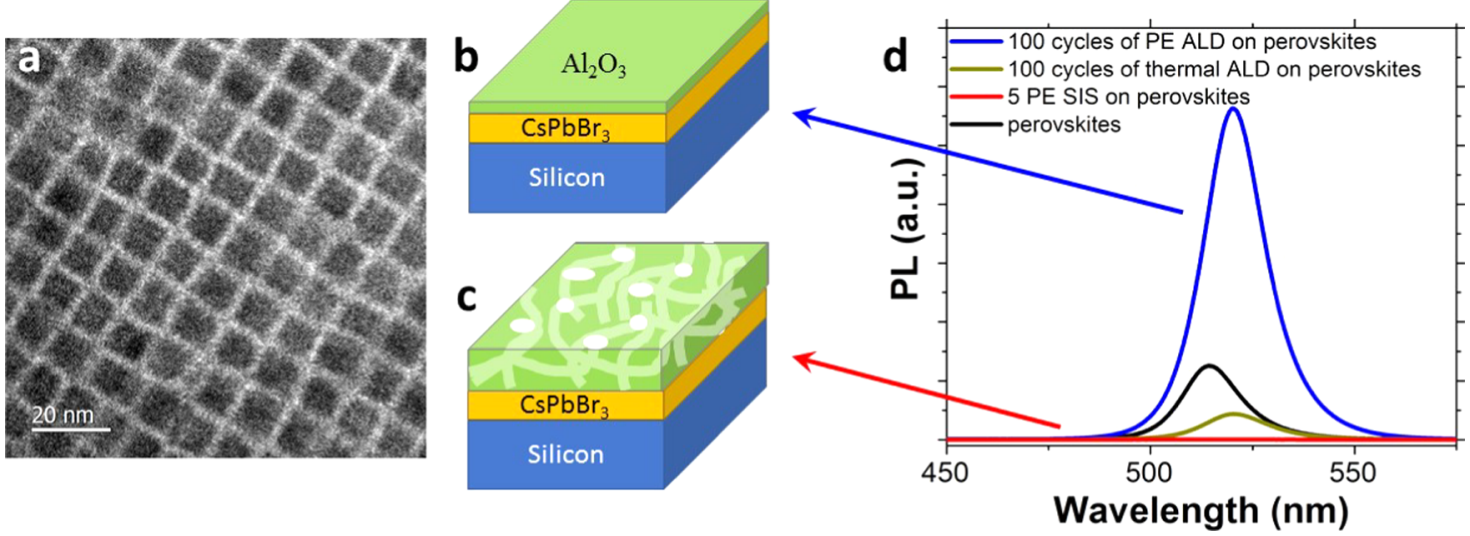

Figure 7. (a) Transmission electron microscopy (TEM) image of cesium lead bromide (CLB) nanoparticles used for photoluminescence tests. (b) Schematic of the protective alumina on top of the NP system is included. (c) Schematic of the highly porous alumina on top of the NP system is included. (d) Photoluminescence data after exposure to humid environment for 5 days for cesium lead bromide perovskite nanoparticles unprotected and covered with alumina deposited by: 100 of PE ALD cycles, 100 of water ALD cycles, and 5 PE SIS of PS- $b$-P4VP. Results indicate higher photoluminescence response for nanoparticles protected with 100 PE ALD.

swelling. Figure 6 summarizes the results for the films grown before and after polymer removal. Swelling produced an $11 \mu \mathrm{m}$ thick polymer film that converted into a $\sim 5 \mu \mathrm{m}$ thick nanoporous alumina structure. We believe that the limitations in thickness are due mostly to the penetration depth for the vapor and mechanical compression of the films after polymer removal.

FTIR measurements on swelled PS- $b$-P4VP block-copolymer templates after alumina infiltration as compared to those on the original polymer film indicated similar trends in BCP subjected to thermal and PE SIS (Figure 2b). However, when compared with nonswelled polymers, changes are significant. Specifically, $\mathrm{CH}$ bending of $\mathrm{CH}_{3}$ and $\mathrm{CH}_{2}$ at 1580, 1500, 1450, and 1410 $\mathrm{cm}^{-136}$ became the dominant mechanism of the alumina growth. Negative features for $\mathrm{CH}$ stretching in normalized FTIR spectra of nonswelled samples suggested the consumption of these groups during SIS. With introduced swelling, they become positive, confirming the increased presence of these groups during swelling. PE SIS partially suppresses their intensity, whereas the very little effect of water SIS indicates slower infiltration process.

To obtain information about the interconnectivity of the pores in alumina deposited by PE SIS, we conducted the following experiment. We deposited $10 \mathrm{~nm}$ alumina on the spin-coated layer of perovskite $\mathrm{CsPbrr}_{3} \mathrm{NPs}(\sim 50 \mathrm{~nm}$ film $)$ on a silicon substrate. $\mathrm{CsPbBr}$ NPs are very promising materials for photovoltaic devices with excellent optoelectronic properties; however, their extremely high moisture sensitivity seriously limits their practical application (Figure 7). Exposure to water results in the immediate degradation of $\mathrm{CsPbBr}_{3} \mathrm{NPs}$ accompanied by complete quenching of PL. The $\mathrm{CsPbr}_{3}$ NP layer with $10 \mathrm{~nm}$ thick alumina film obtained with 100 cycles of PE revealed 2 orders higher PL as compared to that of an unprotected layer of $\mathrm{CsPbBr}$ NPs after 5 day exposure to humid air (40\% humidity). The spin-casted layers of $\mathrm{Cs} \mathrm{PbBr}_{3}$ NPs subjected to water process-based thermal ALD indicated almost complete degradation of perovskite material. In Figure $7 \mathrm{~d}$, the wavelength shift for the samples covered with alumina is observed. This is most likely due to a higher dielectric constant of the medium introduced by the ALD process. ${ }^{41}$

\section{CONCLUSIONS}

We have developed a new synthetic approach for selective sequential infiltration synthesis of nanoporous films using a sequence of exposure of the polymer to TMA vapor and to oxygen plasma. PE SIS offers adventurous rapid infiltration with improved ability to obtain thick films. We show that full infiltration of the polar domain of the BCP template can be achieved within the first cycle, whereas thermal SIS required eight cycles. PE SIS demonstrated high depth of polymer 
infiltration, with up to $5 \mu \mathrm{m}$ thick films being obtained when infiltration of the PS- $b$-P4VP BCP template (75k- $b-25 \mathrm{k})$ was performed after its swelling. Among the methods used for synthesis of porous alumina, PE SIS can be realized at much more gentle conditions (e.g., low temperature and power) and can be potentially applied to the broad range of substrates.

We demonstrated that the solid alumina film protects against water-induced degradation of perovskite NPs. Meanwhile, PE SIS films grown on top of perovskite NPs resulted in immediate degradation of their optical properties upon the water exposure, which indicates high interconnectivity of the porous alumina structure. Such high interconnectivity of nanoporous structures formed by PE SIS can be potentially used for design of highly efficient filtration materials.

In summary, we report on synthesis of highly porous films using the PE ALD process. As compared with thermal ALD, advantages of PE ALD that include higher quality materials, faster deposition rate, deposition of nonstoichiometric materials, and reduced forms of materials and metals lead to our conclusion that PE SIS will improve our ability to synthetize highly porous nanostructured materials for a broad range of applications ranging from catalysis and electrocatalysis to optical coatings.

\section{EXPERIMENTAL METHODS}

Sample Preparation. Block copolymers (BCP) such as poly(styrene-block-4-vinylpyridine) (PS-b-P4VP) with different lengths of polar and nonpolar blocks $\left(\mathrm{PS}_{75 \mathrm{k}}-b-\mathrm{P} 4 \mathrm{VP}_{25 \mathrm{k}}, \mathrm{PS}_{25 \mathrm{k}}-b-\right.$

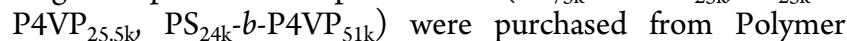
Source, Inc. BCP films of different thicknesses were prepared by spin-coating from 3 and 6 wt \% toluene solutions onto clean silicon substrates with native silicon dioxide films. Cleaning of the substrates was performed as follows: $20 \mathrm{~min}$ of sonication in acetone, followed by $20 \mathrm{~min}$ of sonication in isopropanol, followed by $30 \mathrm{~min}$ of UV ozone exposure. After spin-casting of $\mathrm{BCP}$, the samples were kept on a hot plate at $180{ }^{\circ} \mathrm{C}$ for $10 \mathrm{~min}$ to evaporate residual toluene and to induce microphase separation. Thicknesses of resulting polymer films varied from $100 \pm 10$ to $5000 \pm 40 \mathrm{~nm}$ for 3 and $6 \mathrm{wt} \%$ toluene solutions, respectively. $\mathrm{PS}_{25 \mathrm{k}}-b-\mathrm{P} 4 \mathrm{VP}_{25.5 \mathrm{k}}$ and $\mathrm{PS}_{24 \mathrm{k}}-b-\mathrm{P} 4 \mathrm{VP}_{51 \mathrm{k}}$ polymers were dissolved in dimethylformamide, which is a better solvent for higher concentration of vinylpyridine. Regular poly(methyl methacrylate) (PMMA) 950 polymer of $2 \%$ concentration was purchased from Sigma-Aldrich.

Synthesis of $\mathrm{CsPBr}_{3}$ nanocrystals followed literature procedures. $^{42}$ Lead bromide (>98\%), cesium carbonate (99.9\%), octadecene (90\%), oleylamine (70\%), and oleic acid (90\%) were purchased from Sigma-Aldrich. For synthesis, 69 mg of lead bromide, $0.5 \mathrm{~mL}$ of dry oleylamine, $0.5 \mathrm{~mL}$ of dry oleic acid, and $5 \mathrm{~mL}$ of octadecene were held under vacuum at $120{ }^{\circ} \mathrm{C}$ for $1 \mathrm{~h}$, then heated under nitrogen atmosphere to 170 ${ }^{\circ} \mathrm{C}$. At $170{ }^{\circ} \mathrm{C}, 0.4 \mathrm{~mL}$ of $0.125 \mathrm{M}$ cesium-oleate solution (407 $\mathrm{mg}$ of cesium carbonate, $20 \mathrm{~mL}$ of octadecene, and $1.25 \mathrm{~mL}$ of oleic acid) held at $120{ }^{\circ} \mathrm{C}$ was rapidly injected. The reaction proceeded for $5 \mathrm{~s}$, then was quenched with a water bath. Nanocrystals were purified by centrifugation of the reaction mixture, dispersed in toluene and precipitated with acetonitrile, and re-dissolved in toluene for spin-coating. The sample was characterized by transmission electron microscopy (TEM) using a JEOL-2100F TEM.

The sequential infiltration synthesis reactant, such as trimethylaluminum $\left(\mathrm{Al}\left(\mathrm{CH}_{3}\right)_{3}\right.$, TMA 96\%), was purchased from Sigma-Aldrich and used as received. The high-purity research grade oxygen gas was purchased from Praxair Inc.

Polymer Swelling. Swelling of the polymer films to increase SIS infiltration depth and provide uniformity of the resulting nanoporous films was achieved by immersing the whole sample into pure ethanol and keeping it at $70{ }^{\circ} \mathrm{C}$ for $1 \mathrm{~h}$. The samples were then dried under nitrogen gas flow.

Sequential Infiltration Synthesis. PE SIS was performed using the remote plasma atomic layer deposition (ALD) system, $\mathrm{RMPA}-4000 \mathrm{~L}$. The $\mathrm{Al}_{2} \mathrm{O}_{3}$ coatings were produced by infiltrating the polymer films using binary reactions of TMA/ oxygen plasma. The exposure of BCP films to TMA vapor results in selective binding to polar groups in microphaseseparated polymer domains. Selectively bound $\mathrm{Al}-\left(\mathrm{CH}_{3}\right)_{2}$ converts into $\mathrm{Al}_{2} \mathrm{O}_{3}$ in the subsequent SIS half-cycle of exposure to oxygen plasma. The PE SIS was performed at 90 ${ }^{\circ} \mathrm{C}$ (below the polymer glass transition temperatures) to avoid the flow of swelling-formed, predefined polymer structures. Silicon or glass substrates with polymer films were loaded onto a stainless-steel tray and kept in $200 \mathrm{sccm}$ argon flow for at least 30 min prior to deposition. One cycle of PE SIS was performed as follows: 3 Torr of the synthesis reactant precursor, TMA, was admitted into the reactor using argon carrier gas $(20 \mathrm{sccm})$ for $400 \mathrm{~s}$. Thereafter, the excess of the reactant was evacuated and was followed by admitting 6 Torr of oxygen gas and igniting oxygen plasma with $300 \mathrm{~W}$ power for $20 \mathrm{~s}$; the chamber was then purged with $200 \mathrm{sccm}$ of argon to remove noninfiltrated byproducts. The cycle was repeated several times to determine the degree of infiltration.

Thermal Annealing of the Polymers. Following SIS, the polymer component of the resulting film was removed by baking the samples in a Thermo Fisher Scientific tube furnace at $450{ }^{\circ} \mathrm{C}$ for $2 \mathrm{~h}$ while flowing oxygen gas at $50 \mathrm{sccm}$. Upon cooling, removal of carbon was confirmed with energy dispersive $\mathrm{X}$-ray spectroscopy analysis of the film.

Deposition of $\mathrm{Al}_{2} \mathrm{O}_{3}$ on Perovskite Nanoparticles. Compatibility of the procedure with water-sensitive materials was evaluated by growing aluminum oxide films on cesium lead bromide nanoparticles that were synthesized using a previously described procedure. ${ }^{42}$ Thin films of cesium lead bromide were produced by spin-coating CLB-containing toluene solution on silicon wafers. After drying in a nitrogen atmosphere, the films were exposed directly to ALD procedures with the polymer deposition step omitted. One hundred cycles of PE ALD and thermal ALD procedures were used to coat the perovskite samples with a thin aluminum oxide film.

Characterization. Scanning electron microscopy (SEM) images with energy dispersive spectra analysis were collected with the FEI NOVA 200 system. Small angle X-ray scattering (SAXS) and grazing incidence small angle X-ray scattering (GISAXS) data were collected at Beamline 12-ID-B at the Advanced Photon Source. The X-ray beam (14 keV) was exposed to thin-film samples in both transmission and grazing incidence reflection modes for SAXS and GISAXS measurements, respectively. The scattering data were collected with a Pilatus $2 \mathrm{M}$ detector located about $2 \mathrm{~m}$ away from the samples. Changes in the polymer macromolecules during PE SIS were evaluated using a Nicolet 6700 Fourier Transformation Infrared spectrometer (FTIR) with $600-4000 \mathrm{~cm}^{-1}$ spectral range. Thickness of the films and porosity were measured by the J.A. Woolam M-2000V Automated Angle Ellipsometer. Photoluminescence data were collected in reflection geometry using a $405 \mathrm{~nm}$ PicoQuant laser diode excitation source with emission 
fiber-coupled to a silicon CCD. TEM was performed using a JEOL-2100F microscope.

\section{ASSOCIATED CONTENT}

\section{S Supporting Information}

The Supporting Information is available free of charge on the ACS Publications website at DOI: 10.1021/acsomega.7b01314.

Rupture of aluminum oxide films during oxygen-plasmaassisted $(20 \mathrm{~min})$ polymer removal; dependence of the porosity of synthesized metal oxide films on the polar/ nonpolar domain concentration ratio in the swelled PS- $b$ P4VP polymer; GISAXS data linecut for one PE SISdeposited aluminum oxide in nonswelled PS- $b$-p4VP polymer; GISAXS data analysis (PDF)

\section{AUTHOR INFORMATION}

\section{Corresponding Authors}

*E-mail: shevchenko@anl.gov (E.V.S.).

*E-mail: Diana.Berman@unt.edu (D.B.).

\section{ORCID}

Benjamin T. Diroll: 0000-0003-3488-0213

Byeongdu Lee: 0000-0003-2514-8805

Elena V. Shevchenko: 0000-0002-5565-2060

Diana Berman: 0000-0002-9320-9772

\section{Notes}

The authors declare no competing financial interest.

\section{ACKNOWLEDGMENTS}

This work was performed in part at the University of North Texas's Materials Research Facility. Support from Advanced Materials and Manufacturing Processes Institute (AMMPI) at the University of North Texas is acknowledged. Work at the Center for Nanoscale Materials, Advanced Photon Source, and Electron Microscopy Center was supported by the U.S. Department of Energy, Office of Science, Office of Basic Energy Sciences under Contract No. DE-AC0206CH-11357.

\section{REFERENCES}

(1) Peng, Q.; Tseng, Y.-C.; Darling, S. B.; Elam, J. W. A Route to Nanoscopic Materials via Sequential Infiltration Synthesis on Block Copolymer Templates. ACS Nano 2011, 5, 4600-4606.

(2) Tseng, Y.-C.; Peng, Q.; Ocola, L. E.; Czaplewski, D. A.; Elam, J. W.; Darling, S. B. Etch properties of resists modified by sequential infiltration synthesis. J. Vac. Sci. Technol., B: Nanotechnol. Microelectron.: Mater., Process., Meas., Phenom. 2011, 29, No. 06FG01.

(3) Tseng, Y.-C.; Peng, Q.; Ocola, L. E.; Elam, J. W.; Darling, S. B. Enhanced Block Copolymer Lithography Using Sequential Infiltration Synthesis. J. Phys. Chem. C 2011, 115, 17725-17729.

(4) Ding, S.-J.; Wang, P.-F.; Wan, X.-G.; Zhang, D. W.; Wang, J.-T.; Lee, W. W. Effects of thermal treatment on porous amorphous fluoropolymer film with a low dielectric constant. Mater. Sci. Eng., B 2001, 83, 130-136.

(5) Kim, J. K.; Yang, S. Y.; Lee, Y.; Kim, Y. Functional nanomaterials based on block copolymer self-assembly. Prog. Polym. Sci. 2010, 35, $1325-1349$.

(6) Qi, Z.-M.; Honma, I.; Zhou, H. Nanoporous leaky waveguide based chemical and biological sensors with broadband spectroscopy. Appl. Phys. Lett. 2007, 90, No. 011102.

(7) Buskens, P.; Burghoorn, M.; Mourad, M. C. D.; Vroon, Z. Antireflective Coatings for Glass and Transparent Polymers. Langmuir 2016, 32, 6781-6793.
(8) Guldin, S.; Kohn, P.; Stefik, M.; Song, J.; Divitini, G.; Ecarla, F.; Ducati, C.; Wiesner, U.; Steiner, U. Self-Cleaning Antireflective Optical Coatings. Nano Lett. 2013, 13, 5329-5335.

(9) Kennedy, S. R.; Brett, M. J. Porous broadband antireflection coating by glancing angle deposition. Appl. Opt. 2003, 42, 4573-4579.

(10) Kumeria, T.; Santos, A.; Rahman, M. M.; Ferre-Borrull, J.; Marsal, L. F.; Losic, D. Advanced Structural Engineering of Nanoporous Photonic Structures: Tailoring Nanopore Architecture to Enhance Sensing Properties. ACS Photonics 2014, 1, 1298-1306.

(11) Ju, H.; Zhang, X.; Wang, J. Biosensors Based on Nanoporous Materials. In NanoBiosensing: Principles, Development and Application; Springer: New York, NY, 2011; pp 171-205.

(12) Broom, D. P.; Webb, C. J.; Hurst, K. E.; Parilla, P. A.; Gennett, T.; Brown, C. M.; Zacharia, R.; Tylianakis, E.; Klontzas, E.; Froudakis, G. E.; Steriotis, T. A.; Trikalitis, P. N.; Anton, D. L.; Hardy, B.; Tamburello, D.; Corgnale, C.; van Hassel, B. A.; Cossement, D.; Chahine, R.; Hirscher, M. Outlook and challenges for hydrogen storage in nanoporous materials. Appl. Phys. A 2016, 122, 151.

(13) Xi, J. Q.; Kim, J. K.; Schubert, E. F. Silica nanorod-array films with very low refractive indices. Nano Lett. 2005, 5, 1385-1387.

(14) Xi, J. Q.; Schubert, M. F.; Kim, J. K.; Schubert, E. F.; Chen, M. F.; Lin, S. Y.; Liu, W.; Smart, J. A. Optical thin-film materials with low refractive index for broadband elimination of Fresnel reflection. Nat. Photonics 2007, 1, 176-179.

(15) Zou, L.; Li, X.; Zhang, Q.; Shen, J. An Abrasion-Resistant and Broadband Antireflective Silica Coating by Block Copolymer Assisted Sol-Gel Method. Langmuir 2014, 30, 10481-10486.

(16) Muth, J.; Poggie, M.; Kulesha, G.; Meneghini, R. M. Novel Highly Porous Metal Technology in Artificial Hip and Knee Replacement: Processing Methodologies and Clinical Applications. JOM 2013, 65, 318-325.

(17) Ryan, G.; Pandit, A.; Apatsidis, D. P. Fabrication methods of porous metals for use in orthopaedic applications. Biomaterials 2006, 27, 2651-2670.

(18) Skeldon, P.; Wang, H. W.; Thompson, G. E. Formation and characterization of self-lubricating MoS2 precursor films on anodized aluminium. Wear 1997, 206, 187-196.

(19) Hiratsuka, K.; Asakawa, M.; Funakoshi, A.; Takaya, M. Effect of Impregnation of Iodine Complex on Friction of Anodic Oxide of Aluminum. Tribol. Lett. 2002, 13, 77-80.

(20) Takaya, M.; Hashimoto, K.; Toda, Y.; Maejima, M. Novel tribological properties of anodic oxide coating of aluminum impregnated with iodine compound. Surf. Coat. Technol. 2003, 169$170,160-162$.

(21) Jackson, E. A.; Hillmyer, M. A. Nanoporous membranes derived from block copolymers: from drug delivery to water filtration. ACS Nano 2010, 4, 3548-3553.

(22) Wei, Q.; Xiong, F.; Tan, S.; Huang, L.; Lan, E. H.; Dunn, B.; Mai, L. Porous One-Dimensional Nanomaterials: Design, Fabrication and Applications in Electrochemical Energy Storage. Adv. Mater. 2017, 29, No. 1602300.

(23) Chou, S. Y.; Krauss, P. R.; Renstrom, P. J. Imprint Lithography with 25-Nanometer Resolution. Science 1996, 272, 85-87.

(24) Biswas, M.; Libera, J. A.; Darling, S. B.; Elam, J. W. New Insight into the Mechanism of Sequential Infiltration Synthesis from Infrared Spectroscopy. Chem. Mater. 2014, 26, 6135-6141.

(25) Biswas, M.; Libera, J. A.; Darling, S. B.; Elam, J. W. Kinetics for the Sequential Infiltration Synthesis of Alumina in Poly(methyl methacrylate): An Infrared Spectroscopic Study. J. Phys. Chem. C 2015, 119, 14585-14592.

(26) Peng, Q.; Tseng, Y.-C.; Darling, S. B.; Elam, J. W. Nanoscopic Patterned Materials with Tunable Dimensions via Atomic Layer Deposition on Block Copolymers. Adv. Mater. 2010, 22, 5129-5133.

(27) Suh, H. S.; Kim, D. H.; Moni, P.; Xiong, S.; Ocola, L. E.; Zaluzec, N. J.; Gleason, K. K.; Nealey, P. F. Sub-10-nm patterning via directed self-assembly of block copolymer films with a vapour-phase deposited topcoat. Nat. Nanotechnol. 2017, 12, 575. 
(28) Barry, E.; Mane, A. U.; Libera, J. A.; Elam, J. W.; Darling, S. B. Advanced oil sorbents using sequential infiltration synthesis. J. Mater. Chem. A 2017, 5, 2929-2935.

(29) Berman, D.; Guha, S.; Lee, B.; Elam, J. W.; Darling, S. B.; Shevchenko, E. V. Sequential Infiltration Synthesis for the Design of Low Refractive Index Surface Coatings with Controllable Thickness. ACS Nano 2017, 11, 2521-2530.

(30) Prosek, T.; Taube, M.; Dubois, F.; Thierry, D. Application of automated electrical resistance sensors for measurement of corrosion rate of copper, bronze and iron in model indoor atmospheres containing short-chain volatile carboxylic acids. Corros. Sci. 2014, 87, 376-382.

(31) Ylivaara, O. M. E.; Liu, X.; Kilpi, L.; Lyytinen, J.; Schneider, D.; Laitinen, M.; Julin, J.; Ali, S.; Sintonen, S.; Berdova, M.; Haimi, E.; Sajavaara, T.; Ronkainen, H.; Lipsanen, H.; Koskinen, J.; Hannula, S.P.; Puurunen, R. L. Aluminum oxide from trimethylaluminum and water by atomic layer deposition: The temperature dependence of residual stress, elastic modulus, hardness and adhesion. Thin Solid Films 2014, 552, 124-135.

(32) Profijt, H. B.; Potts, S. E.; van de Sanden, M. C. M.; Kessels, W. M. M. Plasma-Assisted Atomic Layer Deposition: Basics, Opportunities, and Challenges. J. Vac. Sci. Technol., A 2011, 29, No. 050801.

(33) Pinna, N.; Knez, M. Atomic Layer Deposition of Nanostructured Materials; John Wiley \& Sons, 2012.

(34) Ting, Y.-H.; Park, S.-M.; Liu, C.-C.; Liu, X.; Himpsel, F.; Nealey, P. F.; Wendt, A. E. Plasma etch removal of poly (methyl methacrylate) in block copolymer lithography. J. Vac. Sci. Technol., B: Microelectron. Nanometer Struct.-Process., Meas., Phenom. 2008, 26, 1684-1689.

(35) Krishnamoorthy, S.; Hinderling, C.; Heinzelmann, H. Nanoscale patterning with block copolymers. Mater. Today 2006, 9, 40-47.

(36) Choi, H. W.; Woo, H. J.; Hong, W.; Kim, J. K.; Lee, S. K.; Eum, C. H. Structural modification of poly (methyl methacrylate) by proton irradiation. Appl. Surf. Sci. 2001, 169-170, 433-437.

(37) Brinkhuis, R. H. G.; Schouten, A. J. Thin-film behavior of poly(methyl methacrylates). 2. An FT-IR study of Langmuir-Blodgett films of isotactic PMMA. Macromolecules 1991, 24, 1496-1504.

(38) Namouchi, F.; Smaoui, H.; Fourati, N.; Zerrouki, C.; Guermazi, H.; Bonnet, J. J. Investigation on electrical properties of thermally aged PMMA by combined use of FTIR and impedance spectroscopies. J. Alloys Compd. 2009, 469, 197-202.

(39) Potts, S. E.; Dingemans, G.; Lachaud, C.; Kessels, W. M. M. Plasma-enhanced and thermal atomic layer deposition of $\mathrm{Al} 2 \mathrm{O} 3$ using dimethylaluminum isopropoxide, $[\mathrm{Al}(\mathrm{CH} 3) 2(\mu$-OiPr $)] 2$, as an alternative aluminum precursor. J. Vac. Sci. Technol., A 2012, 30, No. 021505

(40) Wang, Y. Nondestructive Creation of Ordered Nanopores by Selective Swelling of Block Copolymers: Toward Homoporous Membranes. Acc. Chem. Res. 2016, 49, 1401-1408.

(41) Buscema, M.; Steele, G. A.; van der Zant, H. S. J.; CastellanosGomez, A. The effect of the substrate on the Raman and photoluminescence emission of single-layer MoS2. Nano Res. 2014, 7, 561-571.

(42) Diroll, B. T.; Nedelcu, G.; Kovalenko, M. V.; Schaller, R. D. High-Temperature Photoluminescence of $\mathrm{CsPbX} 3(\mathrm{X}=\mathrm{Cl}, \mathrm{Br}, \mathrm{I})$ Nanocrystals. Adv. Funct. Mater. 2017, 27, No. 1606750. 